\title{
Neumopericardio por fístula gastropericárdica
}

\author{
A. Ruano Poblador, A. M. Gay Fernández, M. T. García Martínez, L. Galán Raposo, I. Maruri Chimeno, \\ R. Carracedo Iglesias y J. E. Casal Núñez \\ Servicio de Cirugía General y del Aparato Digestivo. Complejo Hospitalario Universitario de Vigo. Hospital Meixoeiro. \\ Vigo
}

\begin{abstract}
RESUMEN
Presentamos el caso de un varón de 56 años que acude a Urgencias por un cuadro de dolor epigástrico irradiado a hombro izquierdo y a cuello. El diagnóstico de pneumopericardio fue realizado tras una radiografía simple de tórax confirmándose una fístula gastropericárdica con la realización de un tránsito con contraste hidrosoluble. Fue tratado quirúrgicamente practicándose gastrectomía total con reconstrucción esofagoyeyunal y drenaje pericárdico, siendo su evolución favorable.
\end{abstract}

Palabras clave: Pneumopericardio. Fístula gastropericárdica.

\begin{abstract}
This is the case of a 56-year-old male who presented at our Emergency Room complaining of severe epigastric pain that radiated towards the left shoulder and neck. Pneumopericardium was diagnosed with plain chest X-rays, which indicated a gastropericardial fistula. A contrast X-ray film was taken using a watersoluble contrast. A full gastrectomy was performed with esophagojejunostomy reconstruction and pericardial drainage, which resulted in a favourable patient outcome.
\end{abstract}

Key words: Pneumopericardium. Gastropericardial fistula.

Ruano Poblador A, Gay Fernández AM, García Martínez MT, Galán Raposo L, Maruri Chimeno I, Carracedo Iglesias R, Casal Núñez JE. Neumopericardio por fístula gastropericárdica. Rev Esp Enferm Dig 2007; 99: 168-171.

\section{INTRODUCCIÓN}

El neumopericardio es una entidad infrecuente que se define como la presencia de contenido gaseoso en la cavidad pericárdica, motivada por el establecimiento de una comunicación entre el pericardio y estructuras vecinas con contenido aéreo, y menos frecuentemente debido a infecciones por gérmenes productores de gas.

Se han comunicado casos de pneumopericardio espontáneo idiopático pero su causa más frecuente es traumática. También existen causas iatrogénicas, secundarias a patologías de órganos vecinos o infecciones primarias del pericardio (1).

Recibido: 25-01-07.

Aceptado: 01-02-07.

Correspondencia: Alejandro Ruano Poblador. Hospital Meixoeiro. Secretaría de Cirugía General. Meixoeiro, s/n. 36200 Vigo, Pontevedra. e-mail: aruanopoblador@yahoo.es
Pretendemos hacer una revisión de esta entidad tras haber tratado a un paciente con pneumopericardio secundario a perforación de víscera hueca.

\section{CASO CLÍNICO}

Varón de 56 años de edad que refiere dolor epigástrico agudo e intermitente de tres días de evolución con irradiación ocasional a cuello, hombro y brazo izquierdo. Ingresa en Urgencias por dolor de localización precordial y de tipo opresivo irradiado a cuello y brazo izquierdo. Entre sus antecedentes personales destacan: gastrectomía Billroth I por ulcus gástrico hace nueve años y reintervención seis meses después por laparocele.

En la exploración física destaca hipotensión (90/60 $\mathrm{mmHg}$ ), taquicardia (110 l.p.m.) e ingurgitación yugular. La exploración por aparatos y sistemas fue normal. 
Analíticamente se aprecia leucocitosis $\left(19.260 / \mathrm{mm}^{3}\right)$ con neutrofilia $(85,9 \%)$, glucemia $224 \mathrm{mg} / \mathrm{dl}$, GOT 65 $\mathrm{UI} / \mathrm{l}, \mathrm{CPK} 218 \mathrm{UI} / \mathrm{ml}, \mathrm{Na}^{+} 130 \mathrm{mEq} / \mathrm{l}$, actividad de protrombina 59\%. El resto de la analítica era normal. En el electrocardiograma se apreció una supradesnivelación del segmento ST en caras inferior y lateral. En la radiografía de tórax se objetiva elevación del hemidiafragma izquierdo y gas en pericardio compatible con pneumopericardio (Fig. 1).

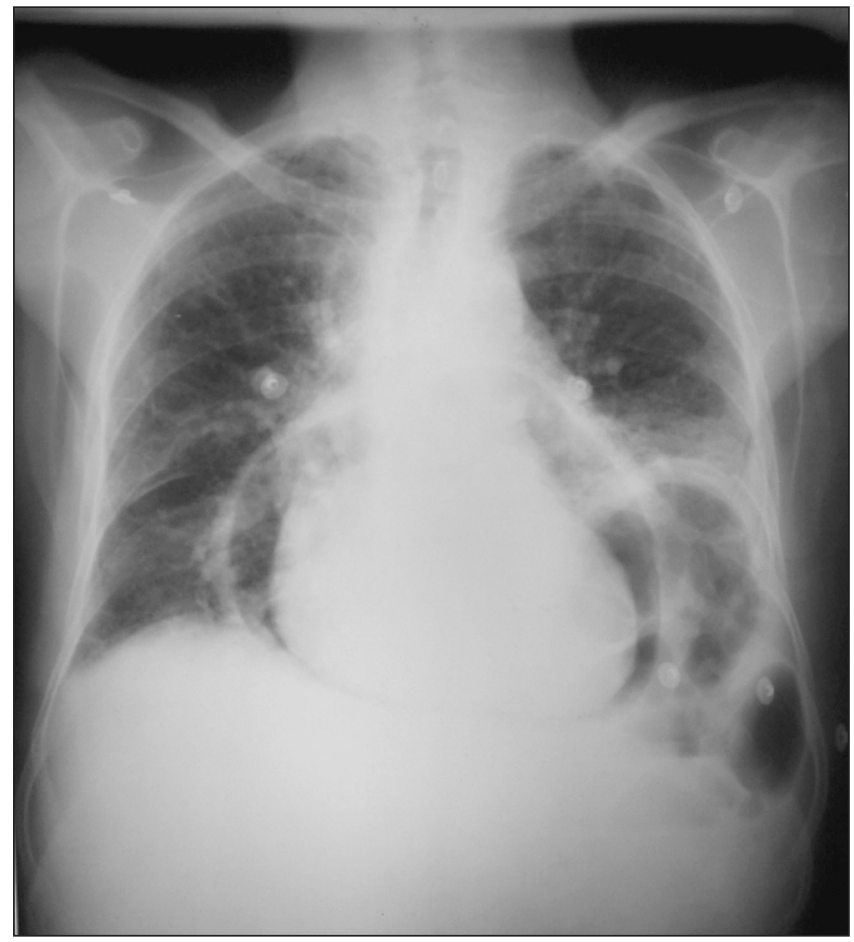

Fig. 1. Pneumopericardio: observamos en la radiografía de tórax una banda de densidad gas rodeando la silueta cardiaca en la cavidad pericárdica.

Con el diagnóstico de presunción de pericarditis aguda el paciente es ingresado en la Unidad de Cuidados Intensivos. Se realiza un estudio gastroduodenal con contraste hidrosoluble objetivándose paso de contraste a cavidad pericárdica (Fig. 2). Es intervenido observándose un diafragma izquierdo elevado con engrosamiento medial en íntimo contacto con fundus gástrico. Se realiza liberación gastro-diafragmática objetivándose una úlcera en fundus gástrico perforada a pericardio. Se aspira líquido de la cavidad pericárdica de aspecto purulento. Se practica gastrectomía total y reconstrucción esofagoyeyunal en Y de Roux transmesocólica, así como biopsia de pared pericárdica. Se deja drenaje pericárdico, así como en cavidades pleural izquierda y abdominal.

En el ecocardiograma postoperatorio se objetiva un mínimo derrame pericárdico y presiones compatibles con

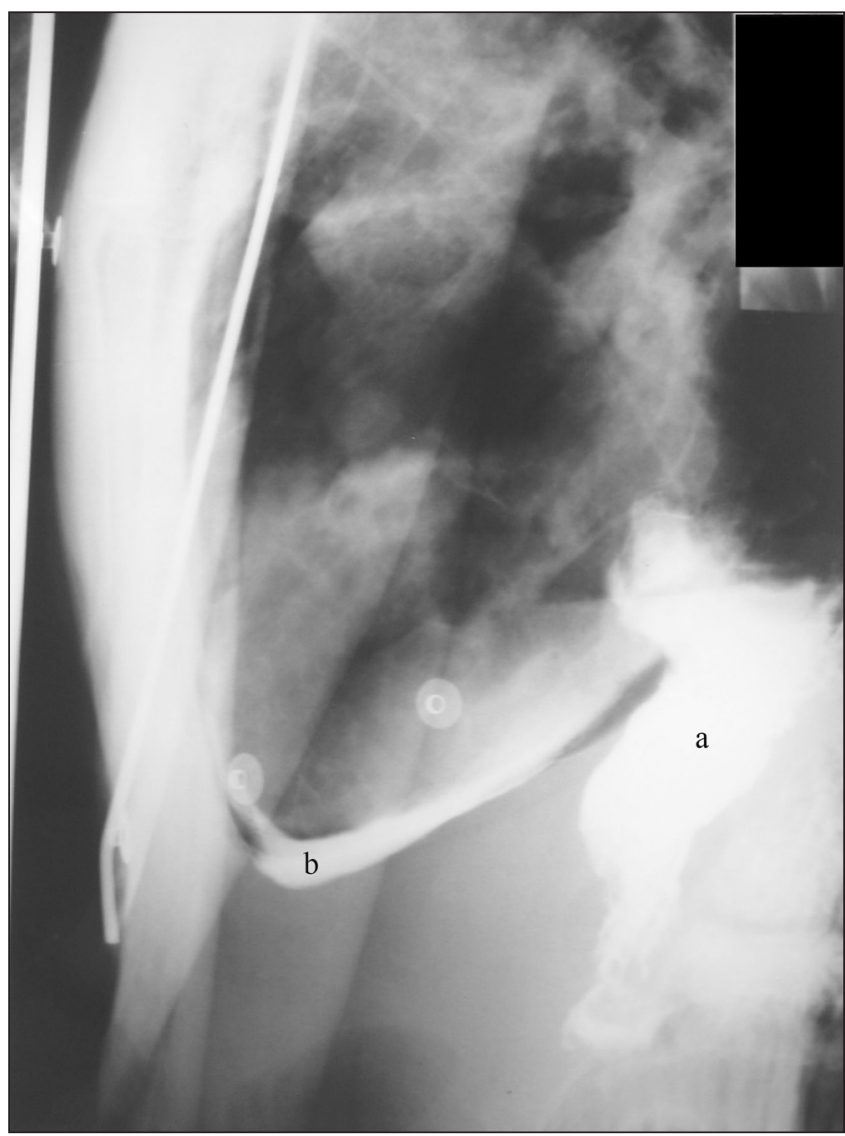

Fig. 2. En el tránsito gastrointestinal con contraste hidrosoluble observamos una fístula gastropericárdica con paso de contraste desde tubo digestivo (a) a cavidad pericárdica (b).

pericarditis restrictiva, pero con buena función ventricular. A los 45 días de la intervención el paciente es reintervenido practicándose colecistectomía por un cuadro clínico de colecistitis aguda.

El informe de anatomía patológica fue de úlcera gástrica perforada con gastritis crónica atrófica moderada con metaplasia intestinal. Tejido pericárdico con fibrosis y parcialmente necrosado observándose glándulas gástricas bien diferenciadas concordantes con mucosa gástrica adherida. Colecistitis aguda.

El paciente fue dado de alta a los tres meses de su primera intervención, permaneciendo asintomático a los nueve años de la misma.

\section{DISCUSIÓN}

El primero en describirlo fue Bricheteau en 1844, que descubrió en un paciente con hidroneumopericardio un signo considerado desde entonces como patognomónico de esta entidad, el "Bruit de Moulin", que consiste en un sonido fluctuante en el precordio (2), aunque sólo suele aparecer en los casos de pneumopericardio complicado. A partir de entonces se han venido publicando casos ais- 
lados en la literatura de distintas etiologías, formas de presentación y tratamiento.

Podemos clasificar la etiología del pneumopericardio en dos grandes grupos: traumáticas y no traumáticas. Las causas de origen traumático son múltiples y variadas, siendo los traumatismos abiertos o cerrados los de mayor incidencia $(1,3)$. Otras etiologías incluyen, ingesta de cuerpos extraños e iatrogénicas como broncoscopia, endoscopia oral, toracocentesis, ventilación mecánica, PAAF, cauterización de membranas esofágicas, intubación endotraqueal, by-pass aortocoronario (1), tras colocación de stent metálico esofágico (4), e incluso tras extracción dental (5). En el caso de las no traumáticas se incluyen asma severo, úlceras esofágicas, neoplasia y rotura espontánea (6) como las más representativas, además de abscesos pulmonares o por tuberculosis, pericarditis por gérmenes productores de gas $(1,3,7)$, abscesos subdiafragmáticos o hepáticos $(8,9)$, cirugía cardiaca reciente, extensión de un pneumomediastino previo (7), rotura alveolar espontánea, pneumopericardio espontáneo idiomático (1) y pseudoquiste pancreático (10). En nuestro caso, el neumopericardio fue debido a una perforación ulcerosa a nivel del fundus gástrico en un paciente con antecedentes de gastrectomía subtotal por úlcera gástrica nueve años antes. El pneumopericardio debido a fístula gastropericárdica por úlcera o carcinoma gástrico es infrecuente, describiéndose únicamente quince casos en la literatura, en esta situación los pacientes suelen tener estómago intratorácico: por hernia de hiato o tras esofagogastrectomía, úlceras con perforación a nivel de región fúndica o pacientes con neoplasia que infiltra el diafragma, especialmente en el síndrome de ZollingerEllison (3).

Estos pacientes suelen presentar dolor torácico penetrante y disnea, siendo característica la irradiación del dolor al hombro izquierdo por irritación pericárdica (11), además pueden presentar fiebre y shock. No obstante, si la causa es por perforación de víscera hueca, podemos observar otros síntomas como disfagia y/u odinofagia, taquicardia, cianosis, hipotensión, distrés respiratorio (7).

Nuestro paciente presentaba dolor epigástrico irradiado a cuello, hombro y brazo izquierdo, hipotensión y taquicardia, siendo diagnosticado de pericarditis aguda.

Como signos clínicos podemos encontrar el ya mencionado "Bruit de Moulin", el timpanismo precordial y la auscultación de sonidos cardiacos metálicos (1).

Shackelford en 1931 emitió un criterio diagnóstico del pneumopericardio: "sonido timpánico agudo a la percusión, un sonido fuerte y metálico sincrónico a los ruidos cardiacos, y nivel líquido aéreo en la cavidad pericárdica en la radiografía de tórax" (12).

Con la radiografía de tórax y el tránsito gastrointestinal con contraste hidrosoluble se diagnostica la causa del pneumopericardio hasta el $70 \%$ de los casos (3), en los casos dudosos el TAC puede ayudar $(3,7)$. En la radiografía de tórax se observa una banda hiperclara que dibuja el corazón (si lo rodea completamente produce el "signo del halo") (7) siendo más específica la banda entre diafragma y corazón (1). Raramente se confina a la región cardiaca, pero no se extiende hacia el mediastino superior o cuello, algo frecuente en el pneumomediastino (7). Si existen dudas, Felson recomienda proyecciones frontales en decúbito lateral derecho e izquierdo con rayo horizontal (1).

Aunque algún autor refiere que la endoscopia puede ser un elemento diagnóstico (3), creemos que debe ser evitada, ya que puede provocar taponamiento cardiaco o empeorarlo si ya está establecido.

El electrocardiograma es inespecífico, ya que puede mostrar cambios compatibles con fibrilación auricular, pericarditis o taponamiento cardiaco, pero puede ser normal en la mayoría de los pacientes $(1,7)$.

En cuanto al ecocardiograma es difícil su interpretación debido a la interposición de aire, existe un signo de orientación diagnóstica llamado "air gap sign" que corresponde a una larga banda de ecos que oscurecen cíclicamente las estructuras cardiacas, aunque este signo no es específico ya que aparece también en el pneumomediastino (1).

A nuestro paciente se le realizó un electrocardiograma donde se observó una supradesnivelación del segmento ST en caras inferior y lateral y en la radiografía de tórax se observa pneumopericardio y elevación del hemidiafragma izquierdo, así, con el diagnóstico de pericarditis el paciente ingresa en la Unidad de Cuidados Intensivos donde debido a la presencia de pneumopericardio se decide realizar un estudio gastroduodenal con contraste hidrosoluble donde objetivamos salida de contraste a cavidad pericárdica y se decide intervención quirúrgica urgente.

Existe constancia de dos pacientes con fístula gastropericárdica que sobrevivieron con tratamiento conservador mediante pericardiocentesis y antibióticos $(13,14)$. No obstante la mejor opción para aumentar la supervivencia en pacientes con esta entidad es un diagnóstico precoz, drenaje pericárdico y cirugía gastrointestinal adaptada a cada caso. En nuestro caso se objetivó una perforación a nivel del fundus gástrico que comunicaba con el pericardio realizándose aspiración del líquido purulento pericárdico, gastrectomía total, esofagoyeyunostomía con reconstrucción en Y de Roux y colocación de drenajes pericárdico, pleural izquierdo y abdominal.

La mortalidad en pacientes con fístula gastropericárdica por úlcera benigna perforada es del $85 \%$ (15). Nuestro paciente fue reintervenido por colecistitis en el postoperatorio y permanece asintomático a los nueve años de su intervención.

\section{BIBLIOGRAFÍA}

1. Muñoz Ávila JA, Jiménez Murillo LM, Montero Pérez FJ, Calderón de la Barca Gázquez JM, Berlango Jiménez A, Durán Serantes M, et al. Neumopericardio: Revisión de la literatura. Revista Clínica Española 1994; 194: 926-8.

2. Bricheteau M. Observation d'hydropneumopericarde accompagne d'un bruit de fluctuation perceptible a l'oreille. Archs Gén Med $1844 ; 4: 334$ 
3. Grandhi TM, Rawlings D, Morrau CG. Gastropericardial fistula: a case report and review of literature. Emerg Med J 2004; 21: 644-5.

4. Dennert B, Ramírez FC, Sanowski RA. Pericardio esophageal fistula associated with metallic stent placement. [abstrad]. Gastrointest Endosc 1997; 45: 82-4.

5. Sandler CM, Libshitz HI, Marks G. Pneumoperitoneum, pneumomediastinum and pneumopericardium following dental extraction [abstrad]. Radiology 1975; 115: 539-40.

6. Ghanem N, Altehoefer C, Springer O, Furtwangler A, Kotter E, Schafer O, et al. Radiological findings in Boerhaave's syndrome. Emerg Radiol 2003; 10: 8-13.

7. Peter Simice MD, Charles V, Zwirewich MD. Gastropericardial fistual complicating benign gastric ulcer: Case report. Can Assoc Radiol J 2000; 51: 244-7.

8. Dorogan DA, Shkola IE, Vinnik IuE. Pneumopericardium as a consequence of a gastro-pericardial anastomosis formed as a result of a subdiaphragmatic abscess. Vestn Rentgenol Radiol 1970; 45: 102-3.
9. Pfaff C, Hunter TB, Silverson ME, Freundlich IM. Pneumopericardium secondary to a fistula. Communication between pericardium and subphrenic-colonic abscess (abstract). JAMA 1976; 235: 2522-3.

10. Balasubramanian P, Jeyamani R, Govil S, Chacko A, Kurian G, Subhash HS, et al. Pancreatico-pericardial fistula: a rare complication of chronic pancreatitis. Indian J Gastroenterol 2004; 23: 31-2.

11. Salling N, Falensteen AM, Larsen LG. Non traumatic perforation of gastric ulcer in a hiatal hernia to the pericardium. Acta Med Scand 1983; 213: 225-6.

12. Shackelford RT. Hydropneumopericardium. JAMA 1931; 96: 187-91.

13. Chapman PR, Boals JR. Pneumopericardium caused by giant gastric ulcer. AJR 1998; 171: 1669-70.

14. Hall MN, Little JM Jr. Penetration of the pericardium by a gastric ulcer-survival after pericardiocentesis. J Am Board Fam Pract 1990; 3: 289-91.

15. Letoquart JP, Fasquel JL, L'Huillier JP, Babatasi G, Gruel Y, Lauvin $\mathrm{R}$, et al. Gastropericardial fistula. Review of the literature apropos of an original case. J Chir (Paris) 1990; 127: 6-12. 